\title{
Prognostic factors for surgically resected non-small cell lung cancer with cavity formation
}

\author{
Shunsuke Shigefuku ${ }^{1}$, Yujin Kudo ${ }^{1}$, Daisuke Yunaiyama ${ }^{2}$, Jun Matsubayashi ${ }^{3}$, Jinho Park ${ }^{2}$, Toshitaka \\ Nagao $^{3}$, Yoshihisa Shimada ${ }^{1}$, Hisashi Saji ${ }^{4}$, Masaru Hagiwara ${ }^{1}$, Tetsuya Okano ${ }^{1}$, Masatoshi Kakihana ${ }^{1}$, \\ Naohiro Kajiwara $^{1}$, Tatsuo Ohira ${ }^{1}$, Norihiko Ikeda ${ }^{1}$
}

${ }^{1}$ Department of Surgery, ${ }^{2}$ Department of Radiology, ${ }^{3}$ Department of Anatomic Pathology, Tokyo Medical University, Tokyo, Japan; ${ }^{4}$ Department of Chest Surgery, St. Marianna University School of Medicine, Kanagawa, Japan

Contributions: (I) Conception and design: S Shigefuku, Y Kudo, Y Shimada, H Saji, N Ikeda; (II) Administrative support: S Shigefuku, Y Kudo, J Park, T Nagao, N Ikeda; (III) Provision of study materials or patients: S Shigefuku, Y Kudo, D Yunaiyama, J Matsubayashi, N Ikeda; (IV) Collection and assembly of data: S Shigefuku, Y Kudo, D Yunaiyama, J Matsubayashi; (V) Data analysis and interpretation: S Shigefuku, Y Kudo, N Ikeda; (VI) Manuscript writing: All authors; (VII) Final approval of manuscript: All authors.

Correspondence to: Yujin Kudo, MD, PhD. Department of Surgery, Tokyo Medical University, 6-7-1 Nishishinjuku, Shinjuku-ku, Tokyo 160-0023, Japan. Email: yjnkudo@gmail.com.

Background: Small pulmonary nodules have been detected frequently by computed tomography (CT). Lung cancers with cavity formation are also easily detected. There are a few reports focused on the cavity wall, although cancer cells exist along the cavity wall, not inside. We evaluated the impact of cavity wall thickness on prognosis and assessed the clinicopathological features in non-small cell lung cancer (NSCLC) with cavity formation.

Methods: Between 2005 and 2011, 1,313 patients underwent complete resection for NSCLC. Of these cases, we reviewed 65 patients $(5.0 \%)$ diagnosed with NSCLC with cavity formation by chest CT. We classified the patients into three groups based on the maximum cavity wall thickness, namely, $\leq 4 \mathrm{~mm}$ (Group 1 , 8 patients), $>4$ and $\leq 15 \mathrm{~mm}$ (Group 2, 33 patients), and $>15 \mathrm{~mm}$ (Group 3, 24 patients).

Results: The number of patients with pathological whole tumor size $>3 \mathrm{~cm}$ was $2(25 \%)$ in Group 1,17 $(52 \%)$ in Group 2, and $23(96 \%)$ in Group $3(\mathrm{P}<0.001)$. Cases with lymph node metastasis were $0(0 \%)$ in Group 1, 5 (15\%) in Group 2, and 10 (42\%) in Group 3 (P=0.016). The 5-year overall survival (OS) rates were $100 \%$ in Group 1, 84.0\% in Group 2, and 52.0\% in Group 3, with significant differences between Group 1 and Group 3 ( $\mathrm{P}=0.044)$ and between Group 2 and Group 3 ( $\mathrm{P}=0.034)$. In univariate analysis, neither whole tumor size nor lymph node metastasis was a prognostic factor for $\mathrm{OS}(\mathrm{P}=0.51, \mathrm{P}=0.27)$. Only cavity wall thickness was a significant prognostic factor by multivariate analysis $(\mathrm{P}=0.009)$.

Conclusions: Maximum cavity wall thickness was an important prognostic factor in NSCLCs with cavity formation, comparable with other established prognostic factors.

Keywords: Cavity wall thickness; non-small cell lung cancer (NSCLC); whole tumor size; prognostic factor; cavity

Submitted Sep 30, 2017. Accepted for publication Jan 09, 2018.

doi: $10.21037 /$ jtd.2018.01.61

View this article at: http://dx.doi.org/10.21037/jtd.2018.01.61 


\section{Introduction}

Lung cancer is one of the leading causes of cancer-related mortality worldwide $(1,2)$, and approximately two-thirds of patients with lung cancer present with locally advanced or metastatic disease at time of diagnosis (3). Early detection of lung cancer is essential to accomplish reduction in mortality. Developments in imaging modalities and the widespread use of low-dose helical computed tomography (CT) for lung cancer have contributed to the increase in detection of small pulmonary lesions (4,5). Primary lung cancers have various appearances in radiological findings, occasionally emerging with cavity formation. The presence of cavities in lung cancer was reported to be a poor prognostic factor for non-small cell lung cancer (NSCLC) (6-8), in addition to pathological stage, which involves tumor differentiation (9), tumor size (10), number of involved lymph nodes (11), and vascular invasion or pleural invasion (12-14). However, the underlying cause of cavity formation accompanying lung cancers as well as why the presence of cavities is likely to be responsible for dismal prognosis remains unclear.

A cavity has been defined in the radiology literature as a gas-filled space, seen as a lucency or low-attenuation area, within pulmonary consolidation, a mass, or a nodule, and sometimes contains fluid (15). This means that a small number of lung cancer cells sometimes occur in "the gasfilled space", but most of the cells lie along the cavity wall. Normal growth pattern in which cancer progression or cell proliferation is directly proportional to increments of tumor size might not apply to the development of cavitary lung cancers. This is because cancer with cavity formation might be caused by bronchial obstruction and a checkvalve mechanism or originate in the wall of a pre-existing cystic space $(16,17)$. In such cases, it remains in doubt that their whole tumor size is associated with malignant potential as in other lung cancers, whereas cavity wall thickness might reflect malignant potential. Our postulates are as follows:

(I) To clarify whether whole tumor size is appropriate as a prognostic factor which determines TNM classification in patients with lung cancers with cavity formation, and;

(II) To evaluate the effect of cavity wall thickness on prognosis in this cohort.

This study was performed to provide pragmatic information for a prognostic factor in lung cancer with cavity formation.

\section{Methods}

\section{Patients}

A total of 1,313 consecutive patients underwent curative operations (lobectomy or bilobectomy with lymph node dissection) for NSCLC at Tokyo Medical University Hospital between January 2005 and December 2011. Patients who had received preoperative chemotherapy, radiotherapy, or both were excluded. Of these cases, 65 patients $(5.0 \%)$ who were diagnosed with NSCLC with a cavity based on chest CT were enrolled. After pulmonary resection, the patients were followed up at 3 to 6 months intervals for 3 years, then at 6 to 12 months intervals for the next 2 years, and thereafter at 1 -year intervals as necessary. The evaluations included physical examination, chest roentgenogram, chest CT, and tumor marker examination. Abdominal CT, brain magnetic resonance imaging, and bone scintigraphy or positron emission tomography CT were performed once a year (with some exceptions) or when recurrence was suspected. The Institutional Review Board of our hospital approved the protocols for data collection and analyses, and waived the need to obtain written informed consent from each patient (No. 2016-224).

\section{Radiological and patbological examinations}

The images from preoperative CT scans were reviewed by two experienced radiologists (Daisuke Yunaiyama and Jinho Park). In this study, cavity formation in lung cancer was defined as an air-containing space greater than $5 \mathrm{~mm}$ in diameter that was located within a tumor and was also surrounded with the wall. The maximum length from the inside wall of a cavity to the margin of the solid part of the tumor in a lung window width and window level was measured as cavity wall thickness. There are no definitive classifications about wall thickness of cavitary lesions. In this study, we used the classification that Woodring et al. developed to predict benign or malignant lesions (18). The patients were classified into three groups based on maximum cavity wall thickness, namely, $4 \mathrm{~mm}$ or smaller (Group 1), both larger than $4 \mathrm{~mm}$ and equal to or smaller than $15 \mathrm{~mm}$ (Group 2), and larger than $15 \mathrm{~mm}$ (Group 3) (Figure $1 A, B, C$ ).

All resected specimens were formalin-fixed and stained with hematoxylin and eosin by routine procedures. All the cases were reviewed by two experienced pathologists (Jun Matsubayashi and Toshitaka Nagao). TNM classification 

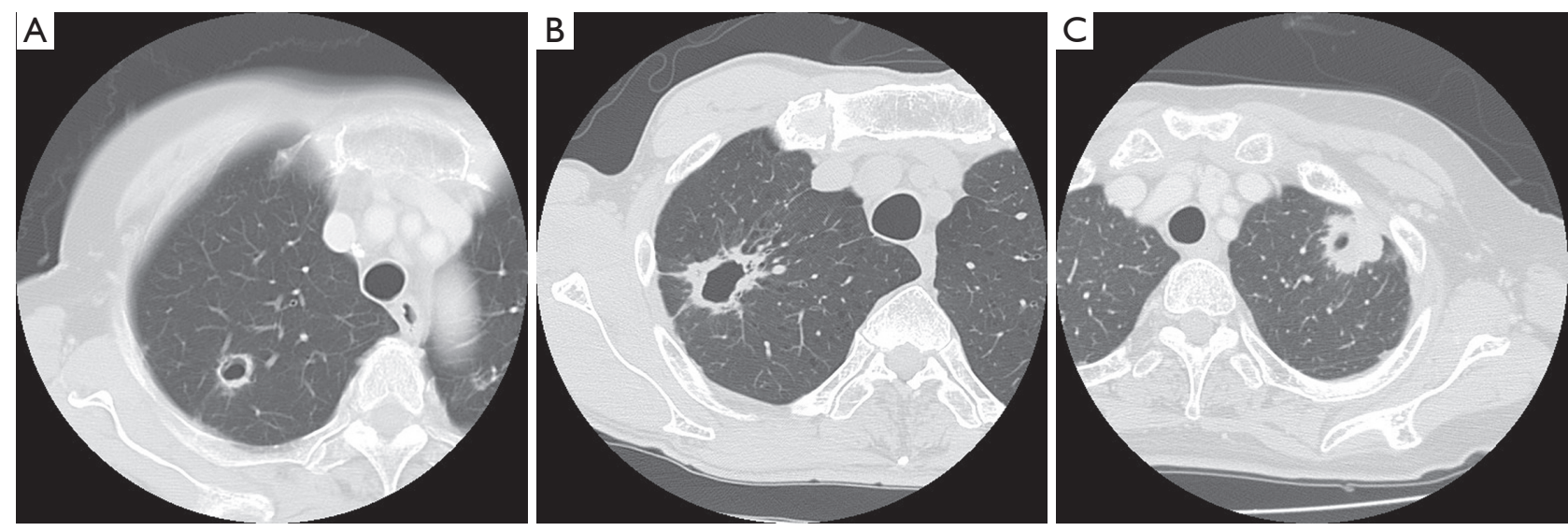

Figure 1 Radiological images of lung cancer with cavity formation. (A) Computed tomography (CT) scan shows a 20 mm-sized nodule with $3.5 \mathrm{~mm}$ of cavity wall thickness located in the right upper lobe (Group 1); (B) CT scan shows a 35 mm-sized tumor with 8.7 mm of cavity wall thickness located in the right upper lobe (Group 2); (C) CT scan shows a $37 \mathrm{~mm}$-sized tumor with $18.8 \mathrm{~mm}$ of cavity wall thickness located in the left upper lobe (Group 3).

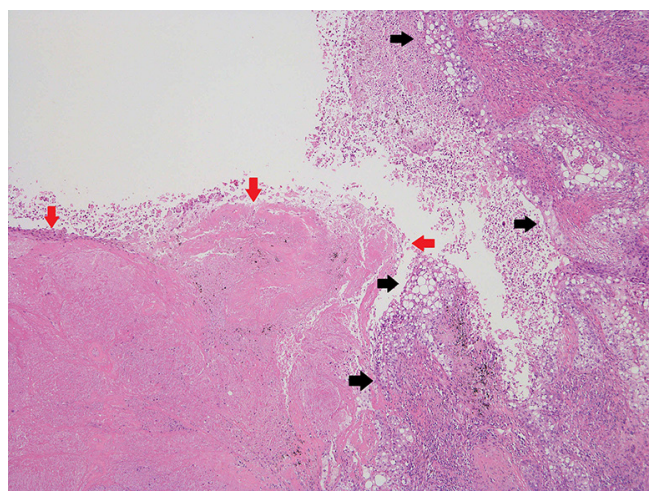

Figure 2 Microscopic examination of the cavity area demonstrates cancer cells present along the cavity wall (black arrows) and necrotic tissue inside of the cavity (red arrows) (hematoxylin-eosin staining; $\times 40)$.

was performed according to the Union for International Cancer Control and the American Joint Committee on Cancer staging system ( $7^{\text {th }}$ edition) (19). Histopathological analyses were performed according to $\mathrm{WHO}$ criteria $\left(4^{\text {th }}\right.$ edition) (20). For detailed examinations of blood vessel invasion (BVI), lymphatic permeation, and visceral pleural invasion (VPI), Elastica van Gieson stain and D2-40 stain were routinely used to evaluate histological structures and tumor invasion. Vascular invasion was defined as the presence of BVI and/or lymphatic permeation. In this study, the presence of necrosis was evaluated in each cavity macroscopically (Figure 2).

\section{Statistical analysis}

Overall survival (OS) and recurrence-free survival (RFS) were estimated using the Kaplan-Meier method, and differences in survival rates were determined by log-rank analysis. OS was defined as the time elapsed from the date of pulmonary resection to the date of the date of death from any cause or last follow-up showing being alive. RFS was defined as the time elapsed from the date of pulmonary resection to the date of the first recurrence or last followup showing no recurrence. The last follow-up observation was censored if the patient was alive or lost to followup. Categorical and continuous variables were analyzed using the Fisher exact test and Student $t$-test, respectively. Multivariate analysis was performed by the backward stepwise procedure for the Cox proportional hazards model. The interaction terms of variables selected in the final model were evaluated by the likelihood ratio test. All tests were two-sided and $\mathrm{P}$ values of less than 0.05 were considered to indicate a statistically significant difference between groups. All statistical calculations were performed using the SPSS statistical software package (version 24.0; SPSS, Inc., Chicago, IL, USA).

\section{Results}

The characteristics of the patients with cavity-containing lung cancer are shown in Table 1. The 65 patients in this study consisted of 48 men (74\%) and 17 women (26\%). 
Table 1 Patient characteristics $(\mathrm{n}=65)$

\begin{tabular}{|c|c|}
\hline Variable & $\begin{array}{c}\text { Number }(\%) \text { or } \\
\text { (range) }\end{array}$ \\
\hline \multicolumn{2}{|l|}{ Sex } \\
\hline Men & $48[74]$ \\
\hline Women & 17 [26] \\
\hline Median age (range), years & 66 [26-82] \\
\hline \multicolumn{2}{|l|}{ Smoking habits } \\
\hline Ever-smoker & 58 [89] \\
\hline Never-smoker & $7[11]$ \\
\hline Operation procedure (lobectomy) & $65[100]$ \\
\hline Median radiological tumor size (range), cm & $3.4(1.5-6.4)$ \\
\hline \multicolumn{2}{|l|}{ Histological type } \\
\hline Adenocarcinoma & $42[64]$ \\
\hline Squamous cell carcinoma & 18 [28] \\
\hline Large cell carcinoma & $2[3]$ \\
\hline Pleomorphic carcinoma & $3[5]$ \\
\hline Median pathological tumor size (range), $\mathrm{cm}$ & $3.5(1.5-7.0)$ \\
\hline \multicolumn{2}{|l|}{ Lymph node metastasis } \\
\hline NO & $50[77]$ \\
\hline $\mathrm{N} 1-2$ & 15 [23] \\
\hline \multicolumn{2}{|l|}{ Pathological stage } \\
\hline I & $38[58]$ \\
\hline II & 20 [31] \\
\hline III & $7[11]$ \\
\hline Vascular invasion (present) & $48[74]$ \\
\hline Visceral pleural invasion (present) & $23[35]$ \\
\hline \multicolumn{2}{|l|}{ Tumor differentiation } \\
\hline Poor/undifferentiated & 16 [25] \\
\hline Well/moderate & 49 [75] \\
\hline \multicolumn{2}{|l|}{ Necrosis in cavity } \\
\hline Present & 32 [49] \\
\hline Absent & $33[51]$ \\
\hline
\end{tabular}

The median age was 66 years (range, $26-82$ years). The median follow-up period was 49.2 months. The median radiological and pathological tumor sizes were 3.4 and $3.5 \mathrm{~cm}$ (range, $1.5-7.0 \mathrm{~cm}$ ), respectively. Histopathological types were adenocarcinoma in 42 patients (64\%), squamous cell carcinoma in 18 patients (28\%), and other types of NSCLC (pleomorphic carcinoma or large cell carcinoma) in 5 patients (8\%). Lymph node metastases were present in 15 patients $(23 \%)$. Pathological findings showed that necrosis in the cavity was present in 32 patients (49\%). Necrosis in the cavity was found in 16 squamous cell carcinomas $(16 / 18,89 \%)$, in 12 adenocarcinomas (12/42, $29 \%)$, and in 4 cases of the other types $(4 / 5,80 \%)$ with a significant difference $(\mathrm{P}<0.001)$.

The associations between cavity wall thickness and clinicopathological factors are shown in Table 2. There were 8 patients (12\%) in Group 1, 33 patients (51\%) in Group 2, and 24 patients (37\%) in Group 3. The number of patients with pathological whole tumor size larger than $3 \mathrm{~cm}$ was $2(25 \%)$ in Group 1, 17 (52\%) in Group 2, and 23 (96\%) in Group $3(\mathrm{P}<0.001)$. A dot plot shows the relationship between cavity wall thickness and whole tumor size in Figure S1. The prevalence of lymph node metastasis was $0 \%$ in Group 1, $15 \%$ in Group 2, and $42 \%$ in Group 3. The prevalence of vascular invasion was $25 \%$ in Group 1, $70 \%$ in Group 2, and 96\% in Group 3. The prevalence of necrosis in cavity of Group 1, Group 2, and Group 3 was $25 \%, 39 \%$, and $71 \%$, respectively. Cavity wall thickness had a statistically significant strong relationship with tumor size $(\mathrm{P}<0.001)$, lymph node metastasis $(\mathrm{P}=0.016)$, stage $(\mathrm{P}=0.001)$, vascular invasion $(\mathrm{P}<0.001)$, VPI $(\mathrm{P}=0.012)$, and necrosis in cavity $(\mathrm{P}=0.022)$. There was no association between cavity wall thickness and histological type.

The 5-year OS rate of Group 1, Group 2, and Group 3 was $100 \%, 84.0 \%$, and $52.0 \%$, respectively. There was a statistically significant difference between Group 1 and Group 3 ( $\mathrm{P}=0.044)$, and between Group 2 and Group 3 $(\mathrm{P}=0.034)$ (Figure $3 A)$. The 5-year RFS rate of Group 1, Group 2, and Group 3 was $100 \%, 75.4 \%$, and $37.0 \%$, respectively. The difference in the RFS rate was statistically significant between Group 1 and Group $3(\mathrm{P}=0.005)$, and between Group 2 and Group $3(\mathrm{P}=0.003)$ (Figure 3B). Considering these results, cavity wall thickness could be recategorized into two groups in the following analysis, namely, $15 \mathrm{~mm}$ or smaller (Group 1 + Group 2) and larger than $15 \mathrm{~mm}$ (Group 3).

Univariate analysis indicated that cavity wall thickness was a statistically significant prognostic factor for OS, and that age, lymph node metastasis, pathological stage, vascular invasion, VPI, tumor differentiation, and cavity wall thickness were significant prognostic factors for RFS 
Table 2 Relationship between cavity wall thickness and other clinicopathological factors $(n=65)$

\begin{tabular}{|c|c|c|c|c|}
\hline Variable & \multicolumn{3}{|c|}{ Cavity wall thickness } & $P$ value \\
\hline Age (years), n [\%] & & & & NS (0.57) \\
\hline$>70$ & $6[75]$ & $23[70]$ & $14[58]$ & \\
\hline$\leq 70$ & $2[25]$ & $10[30]$ & $10[42]$ & \\
\hline Men & $3[38]$ & $25[76]$ & 20 [83] & \\
\hline Women & $5[62]$ & $8[24]$ & $4[17]$ & \\
\hline Smoking history, n [\%] & & & & NS (0.38) \\
\hline Ever-smoker & $6[75]$ & 30 [91] & 22 [92] & \\
\hline Squamous & $2[25]$ & $8[24]$ & 8 [33] & \\
\hline Non-squamous & $6[75]$ & $25[76]$ & $16[67]$ & \\
\hline Pathological tumor size $(\mathrm{cm}), \mathrm{n}$ [\%] & & & & $<0.001^{*}$ \\
\hline$\leq 3$ & $6[75]$ & $16[48]$ & $1[4]$ & \\
\hline$>3$ & $2[25]$ & $17[52]$ & $23[96]$ & \\
\hline Lymph node metastasis, $\mathrm{n}$ [\%] & & & & $0.016^{*}$ \\
\hline No & $8[100]$ & $28[85]$ & $14[58]$ & \\
\hline $\mathrm{N} 1-2$ & 0 & $5[15]$ & $10[42]$ & \\
\hline Absent & $6[75]$ & $10[30]$ & $1[4]$ & \\
\hline Visceral pleural invasion, $\mathrm{n}$ [\%] & & & & $0.012^{*}$ \\
\hline Present & $2[25]$ & $7[21]$ & $14[58]$ & \\
\hline Absent & $6[75]$ & 26 [79] & $10[42]$ & \\
\hline Tumor differentiation, $\mathrm{n}[\%]$ & & & & NS (0.084) \\
\hline Well or moderate & $8[100]$ & 26 [79] & 15 [62] & \\
\hline Poor or undifferentiated & 0 & $7[21]$ & $9[38]$ & \\
\hline Necrosis in cavity, $\mathrm{n}[\%]$ & & & & $0.022^{*}$ \\
\hline Present & $2[25]$ & $13[39]$ & $17[71]$ & \\
\hline Absent & $6[75]$ & 20 [61] & 7 [29] & \\
\hline
\end{tabular}

*, $\mathrm{P}<0.05 . \mathrm{NS}$, not significant. 

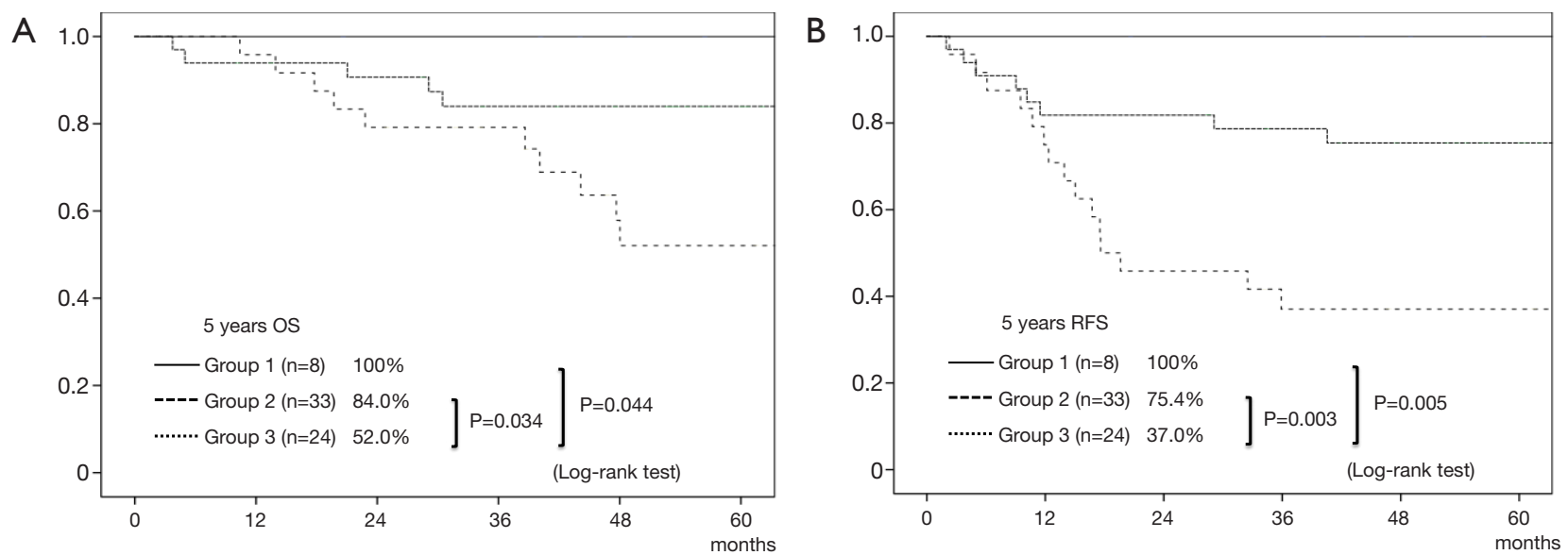

$\begin{array}{lllllll}\text { No. at risk: } & & & & & & \\ \text { Group 1 } & 8 & 8 & 7 & 7 & 5 & 3 \\ \text { Group 2 } & 33 & 30 & 28 & 24 & 22 & 16 \\ \text { Group 3 } & 24 & 23 & 19 & 16 & 10 & 9\end{array}$

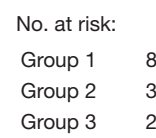

Figure 3 Survival curves in all patients. (A) Overall survival stratified by cavity wall thickness in all patients. The 5 -year OS rate of Group 1, Group 2, and Group 3 was 100\%, 84.0\%, and 52.0\%, respectively. Significant differences in the OS rates were observed between Group 1 and Group $3(\mathrm{P}=0.044)$, and between Group 2 and Group 3 ( $\mathrm{P}=0.034)$. (B) Recurrence-free survival stratified by cavity wall thickness in all patients. The 5-year RFS rate of Group 1, Group 2, and Group 3 was 100\%, 75.4\%, and 37.0\%, respectively. Significant differences in the RFS rates were observed between Group 1 and Group 3 ( $\mathrm{P}=0.005)$, and between Group 2 and Group 3 (P=0.003).

(Table 3). Multivariate analysis showed that cavity wall thickness was a statistically significant prognostic factor for OS [hazard ratio (HR), 3.57; 95\% confidence interval (CI), 1.22-10.48; $\mathrm{P}=0.020$ ] and that age (HR, 3.18; 95\% CI, 1.33-7.62; $\mathrm{P}=0.009)$ and cavity wall thickness (HR, 3.20; 95\% CI, 1.35-7.63; $\mathrm{P}=0.009)$ were significant prognostic factors for RFS (Table 4).

A cohort of the patients without lymph node metastasis showed similar results. The difference in the OS rate was significant between Group 1 and Group $3(\mathrm{P}=0.043)$, and the 5 -year OS rate of Group 2 was worse than that of Group 3, although not statistically significant $(\mathrm{P}=0.052)$ (Figure $4 A)$. Regarding RFS curves, there was a statistically significant difference between Group 1 and Group $3(\mathrm{P}=0.004)$, and between Group 2 and Group $3(\mathrm{P}=0.001)$ (Figure 4B). Multivariate analysis showed that cavity wall thickness was a statistically significant prognostic factor for OS (HR, 4.69; 95\% CI, 1.12-19.69; $\mathrm{P}=0.035)$ and that cavity wall thickness (HR, 5.45; 95\% CI, 1.59-18.64; $\mathrm{P}=0.007$ ) was a significant prognostic factor for RFS (Table S1).

\section{Discussion}

Tumor size, especially solid tumor size, is an important prognostic factor to determine the $\mathrm{T}$ factor of the TNM classification ( $8^{\text {th }}$ edition) in patients with lung cancers (21). In lung cancers with part-solid ground-glass nodules, a solid lesion from radiological findings indicates invasive area of the tumor, and was therefore reported to be associated with prognosis $(22,23)$. There were reports that tumor volume affected the outcome of patients with lung cancer (24). However, lung cancers with cavity formation have less actual tumor volume compared with the whole solid lesion even if the tumor diameter is the same. As mentioned above, it might be unclear whether the whole tumor size is a relevant prognostic factor in lung cancers with cavity formation. Cavity wall thickness may reflect tumor volume with cavity formation.

There were several reports about cavity wall thickness in lung cancer $(18,25)$. Woodring et al. reported that maximum cavity wall thickness provided reliable information to distinguish between benign lesions and malignant lesions (18). Of the lesions $4 \mathrm{~mm}$ or smaller in thickness, $92 \%$ were benign. Of those greater than $15 \mathrm{~mm}, 95 \%$ were malignant. Watanabe et al. analyzed receiver-operating characteristic curves of the cavity wall thickness predicting disease-specific survival, with a cutoff value of $4.5 \mathrm{~mm}$. The surgically resected adenocarcinoma patients with cavity formation were categorized into two groups stratified by the cutoff value of $4 \mathrm{~mm}$ of cavity wall thickness (25). 
Table 3 Univariate analysis of prognostic factors for overall survival and recurrence-free survival

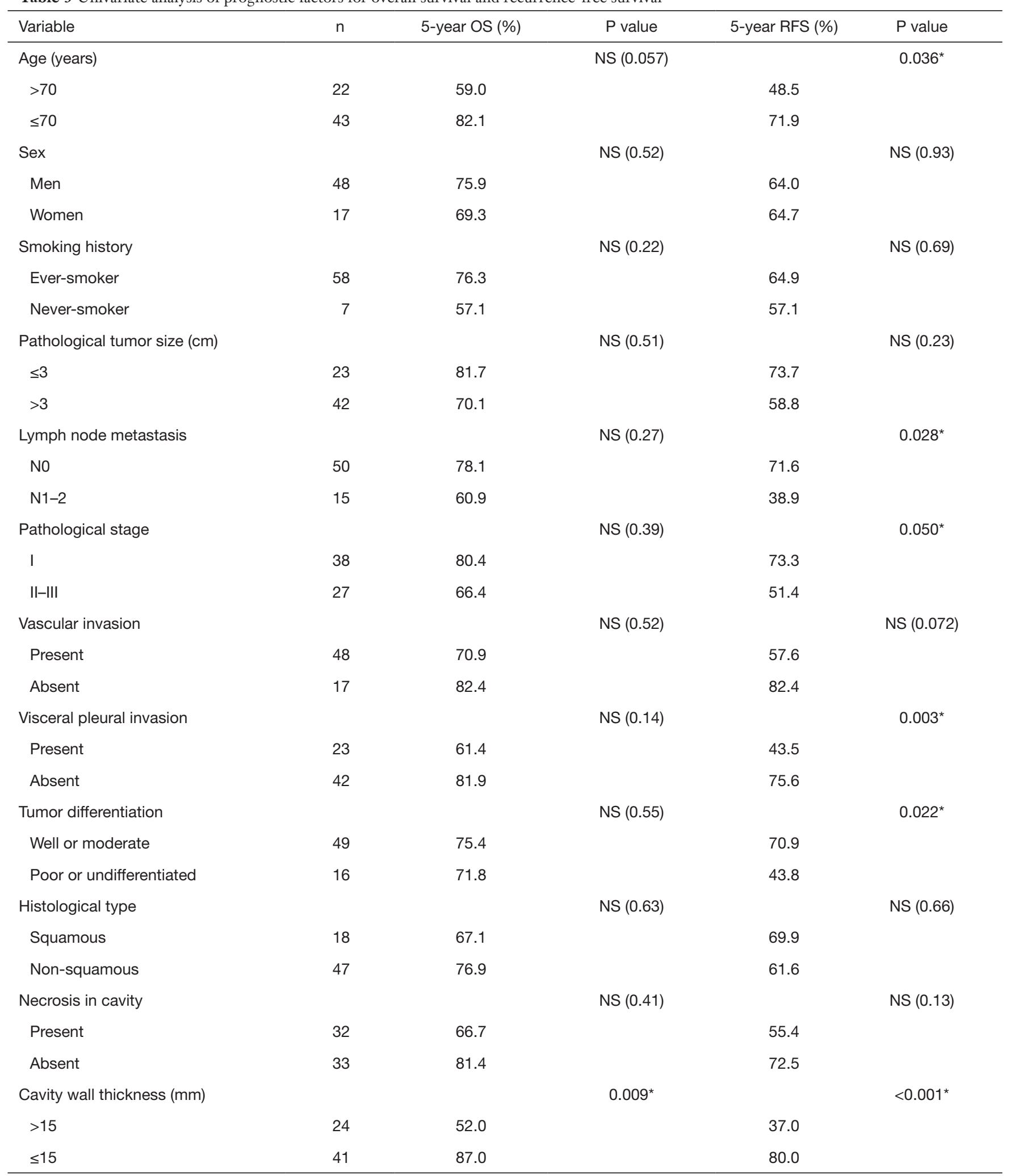

${ }^{*}, \mathrm{P}<0.05$. OS, overall survival; RFS, recurrence-free survival; NS, not significant. 
Table 4 Multivariate analyses of prognostic factors for overall survival and recurrence-free survival

\begin{tabular}{lccc}
\hline Variable & $\begin{array}{c}\text { Hazard } \\
\text { ratio }\end{array}$ & $95 \% \mathrm{Cl}$ & P value \\
\hline OS & & & \\
$\quad \begin{array}{l}\text { Cavity wall thickness } \\
\text { (mm): }>15 \text { vs. } \leq 15\end{array}$ & 3.57 & $1.22-10.48$ & $0.020^{*}$ \\
RFS & & & \\
Age (years): $>70$ vs. $\leq 70$ & 3.18 & $1.33-7.62$ & $0.009^{*}$ \\
$\begin{array}{l}\text { Lymph node metastasis: } \\
\text { N1-2 vs. N0 }\end{array}$ & 2.19 & $0.90-5.33$ & NS $(0.086)$ \\
$\begin{array}{l}\text { Tumor differentiation: poor } \\
\text { or undifferentiated vs. well } \\
\text { or moderate }\end{array}$ & 2.23 & $0.95-5.25$ & NS $(0.065)$ \\
$\begin{array}{l}\text { Cavity wall thickness } \\
\text { (mm): }>15 \text { vs. } \leq 15\end{array}$ & 3.20 & $1.35-7.63$ & $0.009^{*}$ \\
\hline
\end{tabular}

*, $\mathrm{P}<0.05 .95 \% \mathrm{Cl}, 95 \%$ confidence interval; OS, overall survival; RFS, recurrence-free survival; NS, not significant.

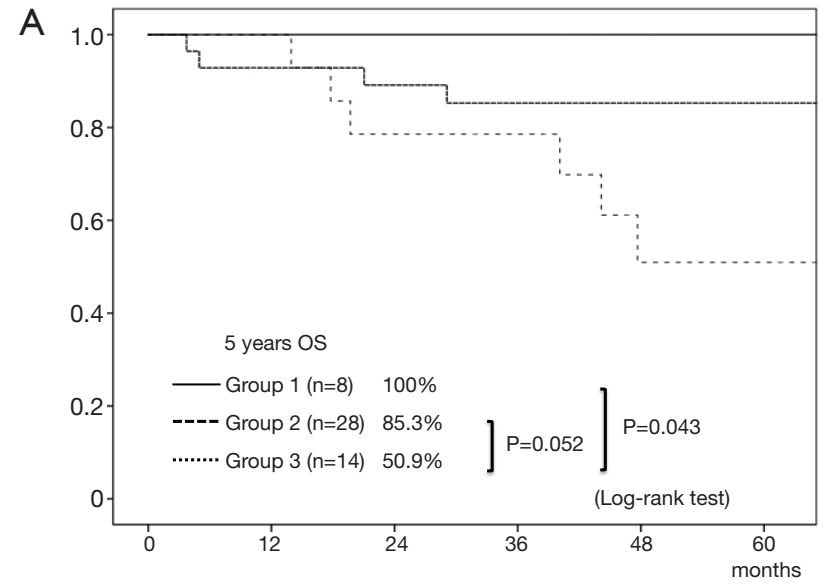

$\begin{array}{lllllll}\text { No. at risk: } & & & & & & \\ \text { Group 1 } & 8 & 8 & 7 & 7 & 5 & 3 \\ \text { Group 2 } & 28 & 25 & 24 & 21 & 19 & 16 \\ \text { Group 3 } & 14 & 14 & 11 & 10 & 5 & 5\end{array}$

The prevalence of lung cancer with cavity formation reported previously was $2-25 \%(16,26-28)$. These variations are explained by the differences in methods of selection. It might depend on time periods in the studies or the prevalence rate of infectious pulmonary diseases during these periods. Our series, which was reviewed by chest CT findings for the patients with surgically resected NSCLC, showed that the prevalence was $5.0 \%$.

Several reports showed that the presence of a cavity was a poor prognostic factor in patients with NSCLC (6-8). Meanwhile, Pentheroudakis et al. reported that there were no statistically significant differences in survival between patients with squamous lung carcinoma with cavity and with solid lesions, but this report included advanced cases that received chemotherapy or radiotherapy (29). Watanabe et al. recently demonstrated that cavity wall thickness was a prognostic factor for cavitary adenocarcinomas, particularly for early stage cancers. The 5 -year OS of stage I patients

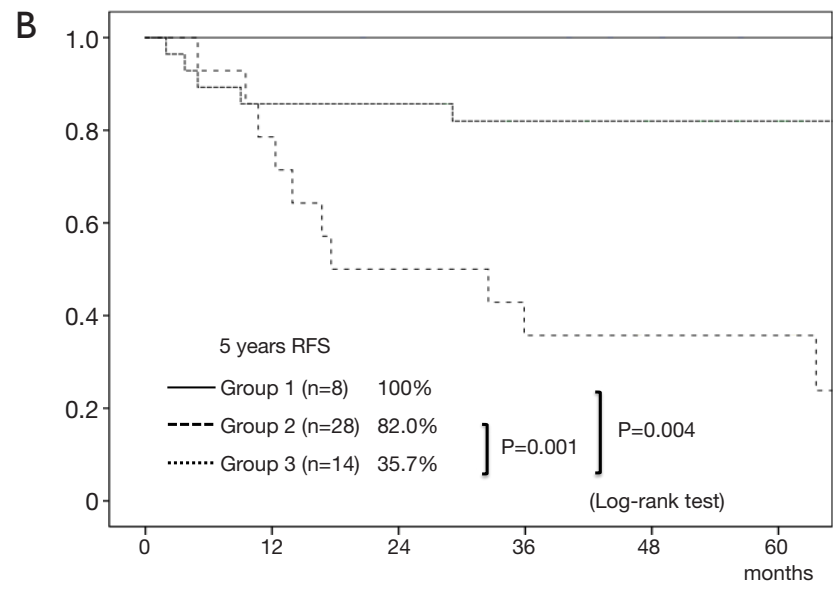

$\begin{array}{lllllll}\text { No. at risk: } & & & & & & \\ \text { Group 1 } & 8 & 8 & 7 & 7 & 5 & 3 \\ \text { Group 2 } & 28 & 24 & 24 & 21 & 19 & 16 \\ \text { Group 3 } & 14 & 11 & 7 & 5 & 4 & 3\end{array}$

Figure 4 Survival curves in the patients without lymph node metastasis. (A) Overall survival stratified by cavity wall thickness in the patients without lymph node metastasis. The 5-year OS rate of Group 1, Group 2, and Group 3 was 100\%, 85.3\%, and 50.9\%, respectively. The difference in the OS rate was significant between Group 1 and Group 3 (P=0.043), and the 5-year OS rate of Group 2 was worse than that of Group 3 ( $\mathrm{P}=0.052)$. (B) Recurrence-free survival stratified by cavity wall thickness in the patients without lymph node metastasis. The 5-year RFS rate of Group 1, Group 2, and Group 3 was 100\%, 82.0\%, and 35.7\%, respectively. Significant differences in the RFS rates were observed between Group 1 and Group 3 ( $\mathrm{P}=0.004)$, and between Group 2 and Group 3 (P=0.001). 
with thin-wall (thickness $\leq 4 \mathrm{~mm}$ ) and thick-wall (thickness $>4 \mathrm{~mm})$ was $91.5 \%$ and $70.1 \%$, respectively $(\mathrm{P}<0.01)(25)$.

In our series, cavity wall thickness had a statistically significant association with prognosis. First, Table 2 shows there were significant relationships both between wall thickness and tumor size, and between wall thickness and lymph node metastasis. Second, Group 1 had $100 \%$ as both OS rate and RFS rate, and Group 3 had the worst prognosis (Figure $3 A, B$ ). However, tumor size and lymph node metastasis were not prognostic factors for $\mathrm{OS}(\mathrm{P}=0.51$ and $\mathrm{P}=0.27$, respectively) in our cases (Table 3). Third, multivariate analysis demonstrated that cavity wall thickness was a prognostic factor for OS and RFS, whereas tumor size, lymph node metastasis, or pathological stage showed no statistical significance (Table 4). Furthermore, the cohort of the patients without lymph node metastasis showed similar results in which cavity wall thickness was also statistically a prognostic factor (Figure $4 A, B$ ). Therefore, our results indicated that cavity wall thickness was most essential for establishing prognosis and treatment strategy in NSCLC patients with cavity formation.

Although the mechanism of cavity formation is often difficult to ascertain, there were explanations of several mechanisms of cavity development in primary lung cancer $(16,17,30)$. The lesion might be caused by necrosis of the primary cancer growth itself, it might arise in the distal portion of the lung by causing bronchial obstruction and a check-valve mechanism or originate in the wall of a preexisting cystic lesion, or it might develop by neoplastic cell autophagy due to a particular enzymatic system which it secreted. The main factors for tumor necrosis were considered to be due to bronchial obstruction and vascular invasion leading to ischemia. In lung adenocarcinoma, recent reports demonstrated that the incidence of vascular invasion was higher in cavitary cases than in non-cavitary cases and that cases with cavity wall thickness larger than $4 \mathrm{~mm}$ had a higher frequency of vascular invasion or necrosis than those with cavity wall thickness $4 \mathrm{~mm}$ or smaller $(7,25)$. Our series showed that $96 \%$ of the patients in Group 3 had vascular invasion and that $71 \%$ of the patients in Group 3 had necrosis in the cavity. Cavity wall thickness was associated with both vascular invasion and necrosis, although there was a difference in prevalence. It was possible that necrosis was not always detected due to washing out during the surgical procedure or when the surgical specimen was prepared for fixation or something else. For the histopathological types, cavity formation in lung cancer was found the most frequently in squamous cell carcinoma $(67-82 \%)$, and was relatively less common in other types of NSCLC $(16,27,31)$. The proportion of squamous cell carcinoma was only $28 \%$ in this study. It might depend on a decrease in typical largesized squamous cell carcinoma with necrosis due to a recent multiplication of small-sized adenocarcinomas.

There were some limitations and biases in the present study, as this is a retrospective analysis in a single institution. The measurement of cavity wall thickness was made by radiological findings, not pathological findings. It was often difficult to measure pathological maximum thickness of the cavity wall accurately, because a sample of cavitary lesion might occasionally collapse and not keep its original configuration during the surgical or pathological procedure, including sample fixation. The pathological thickness could be measured in 61 cases, and there was a positive correlation between radiological thickness and pathological thickness (Figure S2). Even though our series included heterogeneous pathological types of NSCLC, only one of the 14 patients with adenocarcinoma who had recurrence was treated with epidermal growth factor receptor tyrosine kinase inhibitor, which did not significantly affect the above results (data not shown). Nevertheless, our present series revealed highly essential information for NSCLCs with cavities that their wall thickness enabled prognosis rather than tumor size. Multi-institutional studies are mandatory to further validate cavity wall thickness as a prognostic factor for those patients with cavitary-formed NSCLCs due to their relatively low population.

In conclusion, the prevalence of cavity formation in NSCLC was low; however, maximum cavity wall thickness was an important prognostic factor in NSCLCs with cavity formation, comparable with other established prognostic factors. Evaluating wall thickness should help us in deciding on therapeutic options, including minimum resection or conversely extended lymph node dissection. Moreover, we should consider following a more intensive followup schedule to detect their recurrence at the earliest opportunity, basing the evaluation on wall thickness.

\section{Acknowledgements}

We are indebted to the medical editors from the Department of International Medical Communications for editing and reviewing the English manuscript.

\section{Footnote}

Conflicts of Interest: The authors have no conflicts of interest 
to declare.

Ethical Statement: The Institutional Review Board of our hospital approved the protocols for data collection and analyses, and waived the need to obtain written informed consent from each patient (No. 2016-224).

\section{References}

1. Research FfPoC. Cancer Statistics in Japan '15. 2016. Available online: http://ganjoho.jp/data/reg_stat/statistics/ brochure/2015/cancer_statistics_2015.pdf, accessed April 25, 2017.

2. Siegel RL, Miller KD, Jemal A. Cancer Statistics, 2017. CA Cancer J Clin 2017;67:7-30.

3. Rami-Porta R, Bolejack V, Crowley J, et al. The IASLC Lung Cancer Staging Project: Proposals for the Revisions of the T Descriptors in the Forthcoming Eighth Edition of the TNM Classification for Lung Cancer. J Thorac Oncol 2015;10:990-1003.

4. National Lung Screening Trial Research Team, Aberle DR, Adams AM, et al. Reduced lung-cancer mortality with low-dose computed tomographic screening. N Engl J Med 2011;365:395-409.

5. MacMahon H, Naidich DP, Goo JM, et al. Guidelines for Management of Incidental Pulmonary Nodules Detected on CT Images: From the Fleischner Society 2017. Radiology 2017;284:228-43.

6. Gasinska A, Kolodziejski L, Niemiec J, et al. Clinical significance of biological differences between cavitated and solid form of squamous cell lung cancer. Lung Cancer 2005;49:171-9.

7. Watanabe Y, Kusumoto M, Yoshida A, et al. Surgically resected solitary cavitary lung adenocarcinoma: association between clinical, pathologic, and radiologic findings and prognosis. Ann Thorac Surg 2015;99:968-74.

8. Onn A, Choe DH, Herbst RS, et al. Tumor cavitation in stage I non-small cell lung cancer: epidermal growth factor receptor expression and prediction of poor outcome. Radiology 2005;237:342-7.

9. Shimada Y, Saji H, Yoshida K, et al. Pathological vascular invasion and tumor differentiation predict cancer recurrence in stage IA non-small-cell lung cancer after complete surgical resection. J Thorac Oncol 2012;7:1263-70.

10. Suzuki K, Nagai K, Yoshida J, et al. Conventional clinicopathologic prognostic factors in surgically resected nonsmall cell lung carcinoma. A comparison of prognostic factors for each pathologic TNM stage based on multivariate analyses. Cancer 1999;86:1976-84.

11. Saji H, Tsuboi M, Yoshida K, et al. Prognostic impact of number of resected and involved lymph nodes at complete resection on survival in non-small cell lung cancer. J Thorac Oncol 2011;6:1865-71.

12. Shimada Y, Saji H, Kato Y, et al. The Frequency and Prognostic Impact of Pathological Microscopic Vascular Invasion According to Tumor Size in Non-Small Cell Lung Cancer. Chest 2016;149:775-85.

13. Kudo Y, Saji H, Shimada Y, et al. Proposal on incorporating blood vessel invasion into the $\mathrm{T}$ classification parts as a practical staging system for stage I non-small cell lung cancer. Lung Cancer 2013;81:187-93.

14. Kudo Y, Saji H, Shimada Y, et al. Impact of visceral pleural invasion on the survival of patients with non-small cell lung cancer. Lung Cancer 2012;78:153-60.

15. Hansell DM, Bankier AA, MacMahon H, et al. Fleischner Society: glossary of terms for thoracic imaging. Radiology 2008;246:697-722.

16. Chaudhuri MR. Primary pulmonary cavitating carcinomas. Thorax 1973;28:354-66.

17. Farooqi AO, Cham M, Zhang L, et al. Lung cancer associated with cystic airspaces. AJR Am J Roentgenol 2012;199:781-6.

18. Woodring JH, Fried AM, Chuang VP. Solitary cavities of the lung: diagnostic implications of cavity wall thickness. AJR Am J Roentgenol 1980;135:1269-71.

19. Sobin LH, Gospodarowicz MK, Wittekind C. International Union against cancer. TNM classification of malignant tumours. Chichester, West Sussex, UK; Hoboken. NJ: Wiley-Blackwell, 2010.

20. Travis WD, Brambilla E, Burke A, et al. WHO classification of tumours of the lung, pleura, thymus and heart. Lyon, France: IARC, 2015.

21. Brierley J, Gospodarowicz MK, Wittekind C. TNM classification of malignant tumours. Eighth edition. Chichester, West Sussex, UK; Hoboken, NJ: John Wiley \& Sons, Inc., 2017.

22. Travis WD, Asamura H, Bankier AA, et al. The IASLC Lung Cancer Staging Project: Proposals for Coding T Categories for Subsolid Nodules and Assessment of Tumor Size in Part-Solid Tumors in the Forthcoming Eighth Edition of the TNM Classification of Lung Cancer. J Thorac Oncol 2016;11:1204-23.

23. Tsutani Y, Miyata Y, Nakayama H, et al. Prognostic significance of using solid versus whole tumor size on high-resolution computed tomography for predicting pathologic malignant grade of tumors in clinical stage 
IA lung adenocarcinoma: a multicenter study. J Thorac

Cardiovasc Surg 2012;143:607-12.

24. Su XD, Xie HJ, Liu QW, et al. The prognostic impact of tumor volume on stage I non-small cell lung cancer. Lung Cancer 2017;104:91-7.

25. Watanabe Y, Kusumoto M, Yoshida A, et al. Cavity Wall Thickness in Solitary Cavitary Lung Adenocarcinomas Is a Prognostic Indicator. Ann Thorac Surg 2016;102:1863-71.

26. Strang C, Simpson JA. Carcinomatous abscess of the lung. Thorax 1953;8:11-26.

27. Mouroux J, Padovani B, Elkaim D, et al. Should cavitated bronchopulmonary cancers be considered a separate entity? Ann Thorac Surg 1996;61:530-2.

28. Gadkowski LB, Stout JE. Cavitary pulmonary disease. Clin
Microbiol Rev 2008;21:305-33, table of contents.

29. Pentheroudakis G, Kostadima L, Fountzilas G, et al. Cavitating squamous cell lung carcinoma-distinct entity or not? Analysis of radiologic, histologic, and clinical features. Lung Cancer 2004;45:349-55.

30. Omodei Zorini A. Primary carcinomatous cavities of the lung; possible role of neoplastic cell autophagism. Dis Chest 1967;52:329-37.

31. Li BG, Ma DQ, Xian ZY, et al. The value of multislice spiral CT features of cavitary walls in differentiating between peripheral lung cancer cavities and single pulmonary tuberculous thick-walled cavities. Br J Radiol 2012;85:147-52.

Cite this article as: Shigefuku S, Kudo Y, Yunaiyama D, Matsubayashi J, Park J, Nagao T, Shimada Y, Saji H, Hagiwara M, Okano T, Kakihana M, Kajiwara N, Ohira T, Ikeda N. Prognostic factors for surgically resected non-small cell lung cancer with cavity formation. J Thorac Dis 2018;10(2):973-983. doi: $10.21037 /$ jtd.2018.01.61 


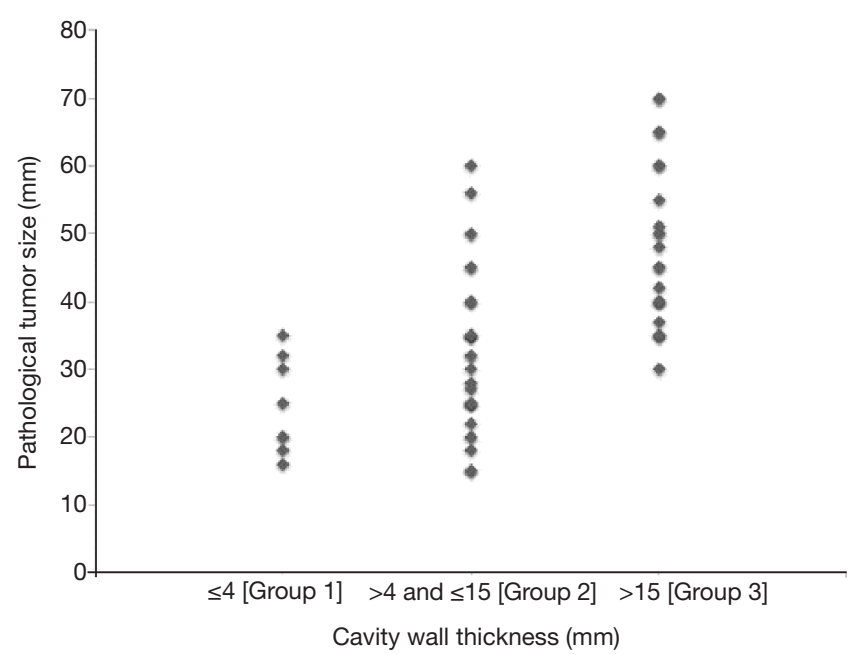

Figure S1 This dot plot shows the relationship between wall thickness and whole tumor size.

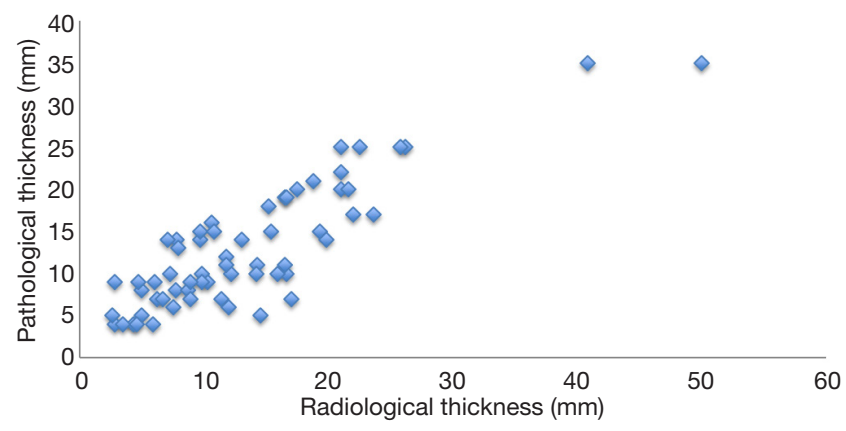

Figure S2 The relationship between pathological thickness and radiological thickness in cavities (61 cases). There was a positive correlation between pathological thickness and radiological thickness. The R-squared value was 0.87 .
Table S1 Multivariate analyses of prognostic factors for overall survival and recurrence-free survival in the patients without lymph node metastasis

\begin{tabular}{|c|c|c|c|}
\hline Variable & Hazard ratio & $95 \% \mathrm{Cl}$ & $P$ value \\
\hline \multicolumn{4}{|l|}{ OS } \\
\hline $\begin{array}{l}\text { Cavity wall thickness } \\
(\mathrm{mm}):>15 \text { vs. } \leq 15\end{array}$ & 4.69 & $1.12-19.69$ & $0.035^{*}$ \\
\hline \multicolumn{4}{|l|}{ RFS } \\
\hline Age (years): $>70$ vs. $\leq 70$ & 2.98 & $0.87-10.17$ & 0.081 \\
\hline $\begin{array}{l}\text { Cavity wall thickness } \\
(\mathrm{mm}):>15 \text { vs. } \leq 15\end{array}$ & 5.45 & $1.59-18.64$ & $0.007^{*}$ \\
\hline
\end{tabular}

